\title{
Function-Orientated Structural Analysis of the Proximal Human Femur
}

\author{
Tobias P. Skuban $^{\mathrm{a}}$ Tobias Vogel $^{\mathrm{c}} \quad$ Andrea Baur-Melnyk ${ }^{\mathrm{b}}$ Volkmar Jansson ${ }^{\mathrm{a}}$ \\ Bernhard Heimkes ${ }^{a}$ \\ Departments of a Orthopedic Surgery and ${ }^{b}$ Radiology, Grosshadern Medical Center, Ludwig-Maximilians-University \\ Munich, Munich, and ' Department of Orthopedic Surgery, St. Josef Hospital, Ruhr University Bochum, Bochum, \\ Germany
}

\section{Key Words}

Biomechanics $\cdot$ Compressive stress $\cdot$ Femur, proximal $\cdot$

Tensile forces $\cdot$ Trabecular system $\cdot$ Trajectories

\begin{abstract}
In his model of the biomechanics of the proximal human femur, Friedrich Pauwels assumes a resultant force acting on the femoral head that is created by the partial body weight and the force of the abductor muscles inserting at the greater trochanter. This model suggests a tensile force in the region of the greater trochanter. An exact examination of the muscle insertions at the greater trochanter resulted in a contrasting hypothesis assuming a local compression stress in the region of the greater trochanter. The aim of this study was to examine which hypothesis is favored by the internal architecture of the proximal femur. Based on the architectural software Allplan ${ }^{\circledR}$, we performed an extended analysis of the trabecular structure within the proximal femur using CT scans of 10 human cadaver femora altogether. According to our results, both the medial and the trochanteric trabecular systems are orientated approximately perpendicular to the arcuate trabecular system [angles between systems ranging from 84.6 to $93.0^{\circ}$ (mean angle $90.7^{\circ}$ ) and from 80.9 to $86.5^{\circ}$, (mean angle $84.9^{\circ}$ ), respectively]; furthermore, the medial trabecular system is orientated perpendicular to the epiphysis of the femoral head (mean of angles: $94.7^{\circ}$ ). The
\end{abstract}

biomechanical interpretation of these results strongly supports the idea of compressive stress in the region of the greater trochanter and makes a predominant tensile force of the abductor muscles highly unlikely.

Copyright $\odot 2009$ S. Karger AG, Basel

\section{Introduction}

In 1935, Friedrich Pauwels developed a still widely accepted model of the force pattern of the human hip joint [Pauwels, 1935]. In this 'classic' model, he sets up the hypothesis that the proximal femur is stressed by a resultant force created by the partial body weight and the antagonistic action of the abductor muscles attached to the greater trochanter. This resultant force directly acts through the center of rotation of the hip joint, which approximately corresponds to the center of the femoral head, resulting in tensile stress of the greater trochanter and bending of the femoral shaft [Pauwels, 1954]. Even today, a multitude

\begin{tabular}{ll}
\hline Abbreviations used in this paper \\
\hline $\mathrm{CV}$ & coefficient of variation \\
SD & standard deviation
\end{tabular}

Prof. Dr. Bernhard Heimkes, Department of Orthopedic Surgery

Grosshadern Medical Center, Ludwig-Maximilians-University Munich

Marchioninistrasse 15, DE-81377 Munich (Germany)

Tel. +4989709539 20, Fax +498970953934

E-Mail Bernhard.Heimkes@med.uni-muenchen.de 


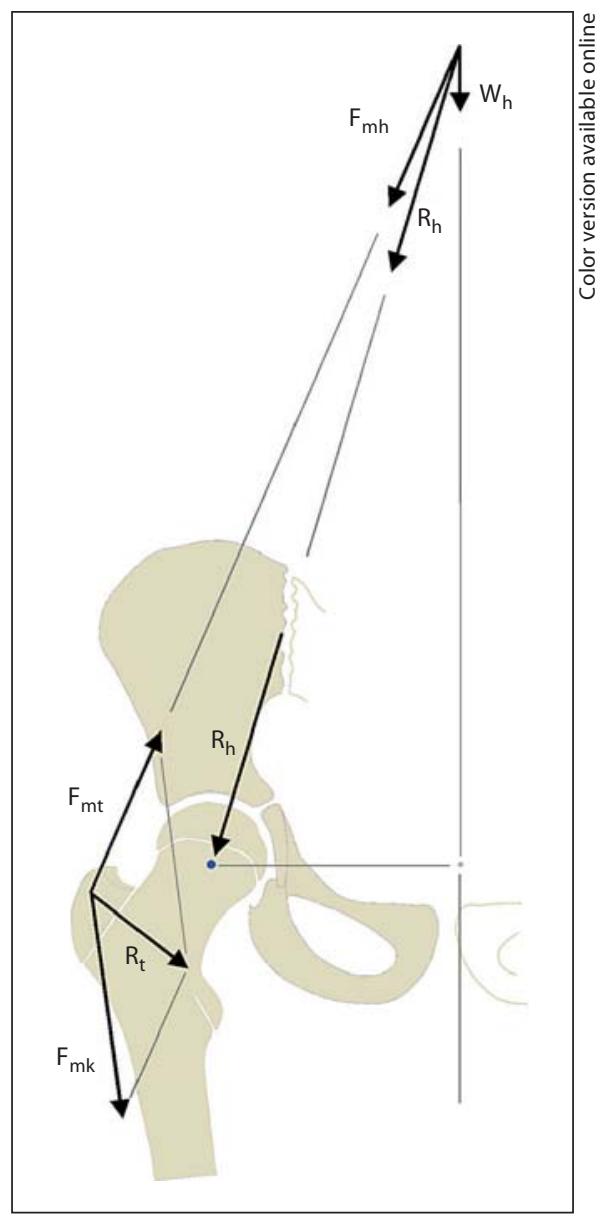

Fig. 1. The proximal femur is stressed by two resultant forces, $R_{h}$ and $R_{t}$. The trochanteric resultant force $R_{t}$ is the vectorial sum of the muscle resultant force $\mathrm{F}_{\mathrm{mt}}$ exerted by all the abductor muscles and the force $F_{m k}$. The force $F_{m k}$ is composed of the traction on the iliotibial tract and the force exerted by the knee extensors, which are connected by the vastus lateralis muscle to the greater trochanter apophysis [Heimkes et al., 1993].

of biomechanical studies designed to test preclinical hip prostheses are based on Pauwels' assumptions [Gebauer et al., 1989; McKellop et al., 1991; Berzins et al., 1993; Buhler et al., 1997; Pedersen et al., 1997; Gotze et al., 2002; Maher and Prendergast, 2002]. Although Pauwels' prediction that the femoral neck would be predominately subjected to bending stress has been repeatedly challenged [Hêrt, 1994; Taylor et al., 1996; Rudman et al., 2006] and various investigators call for the additional consideration of other muscle groups that are acting on the hip joint in order to improve the simulation of the physiological situation when performing biomechanical analyses [Duda et al., 1998; Szivek et al., 2000], Pauwels' model continues to be a more or less unquestioned and accepted working scheme broadly influencing biomechanical concepts and experimental protocols [Carter et al., 1989; Turner et al., 1997; Bergmann et al., 2001; Cristofolini et al., 2007]. However, Heimkes et al. [1993] set up another model of human hip joint biomechanics (fig. 1) following an extensive investigation of the anatomic situation at the greater trochanter: the consideration of the position of the apophyseal plate at the greater trochanter on the one hand and the exact location of the muscle insertion of the abductors and the femoral knee extensors (predominantly the vastus lateralis muscle) on the other resulted in an amendment of the classic model of Pauwels by postulating a second resultant force that is orientated perpendicular to the apophyseal plate [Heimkes et al., 1993]. Regarding this concept, a local compression stress can be assumed in the region of the trabeculae of the greater trochanter, which are traditionally interpreted as tensile trajectories.

An exact knowledge of joint biomechanics is the basis for an understanding of the function of healthy or pathologically altered joints, questions of artificial replacement or a sensitive development of rehabilitation programs [Brand et al., 1994]. Hence, this study was performed in order to compare the models of Pauwels and Heimkes, and to examine which reflects the physiological situation most appropriately.

To arrive at an accurate decision on these biomechanical concepts, the forces acting on the musculoskeletal system have to be measured. Therefore, several approaches have been developed: direct measurement by instrumented prostheses [Bergmann et al., 2001] can be useful to evaluate the resultant force acting on the femoral head; however, the force pattern distal to the femoral head, which may effect the biomechanical situation of the proximal femur, is neglected. Other forces directly affecting the proximal femur (e.g. at the greater trochanter or the femoral shaft) cannot be considered. Furthermore, only patients suffering from musculoskeletal pathologies are provided with these prostheses, which could also have an impact on the biomechanics of the joint studied [Brand et al., 1994]. Furthermore, anatomic structures are affected during the implantation process, which could effect the biomechanical situation. The interpretation of strain gage data [Mikic and Carter, 1995; Aamodt et al., 1997] underlies comparable limitations. Radiographic measurements of stressed skeletal structures, e.g. human femora in the one-legged stance [Taylor et al., 1996], facilitate indirect estimations of the acting forces, but they are too imprecise to develop sophisticated biomechanical concepts. Direct simulation protocols using human ca- 
daver bones, artificial devices or even computerized models (finite element method) are of restricted explanatory power due to the fact that the results are highly dependent on the presumed and often inaccurate external forces acting on the finite element model. Hence, muscle forces could be under- or overestimated, or anatomical structures of biomechanical importance could even be neglected. Furthermore, a detailed representation including all characteristics of the anisotropic trabecular architecture by finite element methods is of overwhelming complexity and could not be achieved yet.

In this study, a completely different approach to inferring a force distribution pattern on the human proximal femur has been applied: since the internal trabecular system of bone tissue aligns with the principal stresses, a widely accepted concept [Pauwels, 1954; Rydell, 1973; Osborne et al., 1980; Fyhrie and Carter, 1986; Kerr et al., 1986; Biewener et al., 1996; Cody et al., 1996; Smit et al., 1997; Turner et al., 1997; Uchiyama et al., 1999; Birnbaum et al., 2001; Gomberg et al., 2003; Ruimerman et al., 2003; Kinney et al., 2005], analysis of this internal bone structure may help to clarify the direction of forces acting on bone tissue. In this way, bone itself can serve as a record of its biomechanical history [Carter et al., 1989] and can provide a basis to examine which of the two biomechanical models in question reflects the physiologic situation most appropriately.

\section{Materials and Methods}

\section{Nomenclature}

According to Martens et al. [1983] and corresponding to the trabecular system firstly described by Meyer [1867] in the 19th century and by Singh et al. [1970] in the 20th century, the internal trabecular structure of the proximal femur is regarded as being composed of three main trabecular systems: the medial system arising from the medial cortex of the femoral shaft extending to the cranial aspect of the femoral head, the trochanteric system arising from the medial cortex of the shaft below the trabeculae of the medial system arising cranially and laterally towards the greater trochanter and the arcuate system beginning in the inferior cortex of the femoral head extending in a curved course through the femoral neck to the lateral cortex of the shaft, therewith overlapping with the trabeculae of the medial and trochanteric system.

\section{Specimens}

In this study, 10 well-conserved left human cadaver femora from adult donors were investigated (for donor and specimen data, see table 1). None showed any pathologic changes, e.g. fractures or signs of arthritis, all were of comparable size and had a centrum-collum-diaphysis angle, i.e. the angle between the longitudinal axis of the femur shaft and that of the femur neck, according to age.
Table 1. Demographic characteristics of the donors and centrumcollum-diaphysis (CCD) angles of the femur specimens

\begin{tabular}{lllllll}
\hline $\begin{array}{l}\text { Specimen } \\
\text { No. }\end{array}$ & Sex & $\begin{array}{l}\text { Age } \\
\text { years }\end{array}$ & $\begin{array}{l}\text { kg } \\
\text { kght }\end{array}$ & $\begin{array}{l}\text { Height } \\
\mathrm{cm}\end{array}$ & Cause of death & $\begin{array}{r}\text { CCD } \\
\text { angle }\end{array}$ \\
\hline 1 & male & 78 & 80 & 174 & heart failure & $130^{\circ}$ \\
2 & female & 80 & 60 & 166 & heart failure & $130^{\circ}$ \\
3 & female & 70 & 64 & 160 & stroke & $128^{\circ}$ \\
4 & male & 68 & 81 & 170 & unknown & $124^{\circ}$ \\
5 & female & 86 & 78 & 168 & heart failure & $127^{\circ}$ \\
6 & female & 77 & 63 & 163 & colon perforation & $139^{\circ}$ \\
7 & male & 71 & 79 & 172 & aortic dissection & $119^{\circ}$ \\
8 & female & 74 & 68 & 164 & heart failure & $125^{\circ}$ \\
9 & female & 49 & 71 & 168 & heart failure & $134^{\circ}$ \\
10 & male & 65 & 82 & 175 & unknown & $127^{\circ}$ \\
\hline & & & & & &
\end{tabular}

\section{CT Scans}

Using a laser level (Stabila 80 LMX-P + L), i.e. a spirit level with built-in laser beam, all femora were positioned in such a way that the plane defined by the main axes of the femoral neck and the shaft were parallel to the horizontal plane (i.e. $0^{\circ}$ anteversion) to avoid projection errors in the subsequent analysis procedure. CT scans were performed using the multislice CT scanner Somatom Sensation 64 (Siemens, workplace UB 30B; field of view $500 \mathrm{~mm}$, resolution $0.4 \times 0.4 \times 0.4 \mathrm{~mm})$. The axial CT slices had a scanto-scan distance of $0.1 \mathrm{~mm}$ and a scan thickness of $0.4 \mathrm{~mm}$; a coronal reconstruction was performed with a reconstructed scanto-scan distance of $0.1 \mathrm{~mm}$ and a reconstructed scan thickness of $0.4 \mathrm{~mm}$. A series of five CT scans from the central portion of each femur with well-identifiable trabecular structures was used for the analysis. Thus, $50 \mathrm{CT}$ scans have been analyzed altogether.

\section{Approximation of the Arcuate Trabecular System}

The aim of this study was to determine the exact course of the three trabecular systems of the proximal human femur in order to use this as a basis for subsequent biomechanical interpretation. Due to the multitude of individual trabeculae giving rise to a high amount of options, an extraction of the exact course of the trabecular system by pure visual judgment may lead to highly inaccurate results. Hence, a more precise method was developed. Allplan ${ }^{\circledR}$ (version 2005; Nemetschek Allplan), an object-oriented computer-aided design software originally developed for architectural purposes, enables an extended analysis of each single CT scan. The program is easy to use and offers the opportunity to perform any graphic construction needed for our analysis. Furthermore, different construction layers could be defined, which could be superimposed in any combination due to a common coordinate system of these layers. Consequently, construction elements, which were not contributory at certain points of the analysis process (e.g. construction lines), could be eradicated, thus better visualizing the construction and avoiding analysis errors. Each CT scan had to be converted into a JPG format for use with Allplan. Every individual trabecula of the three trabecular systems was then marked via the drawing function of Allplan. All marked trabeculae of the arcuate system were afterwards interpreted as a set of straight lines, which was examined in single sections, each 
Fig. 2. All CT scans were analyzed in sections $1 \mathrm{~cm}$ in width beginning at the very medial cortex margin of the femoral head (1-8 in the scan depicted); the cranial and caudal outline of the arcuate system is indicated by two series of straight lines; the arcuate system itself was examined in a cranial and a caudal half (intermediate series of straight lines divides each section in a cranial and a caudal part). Short lines indicate identified trabeculae of the arcuate trabecular system.

Fig. 3. All mediocaudally orientated angles between single trabeculae and the vertical were measured (three angles are depicted in this scheme).

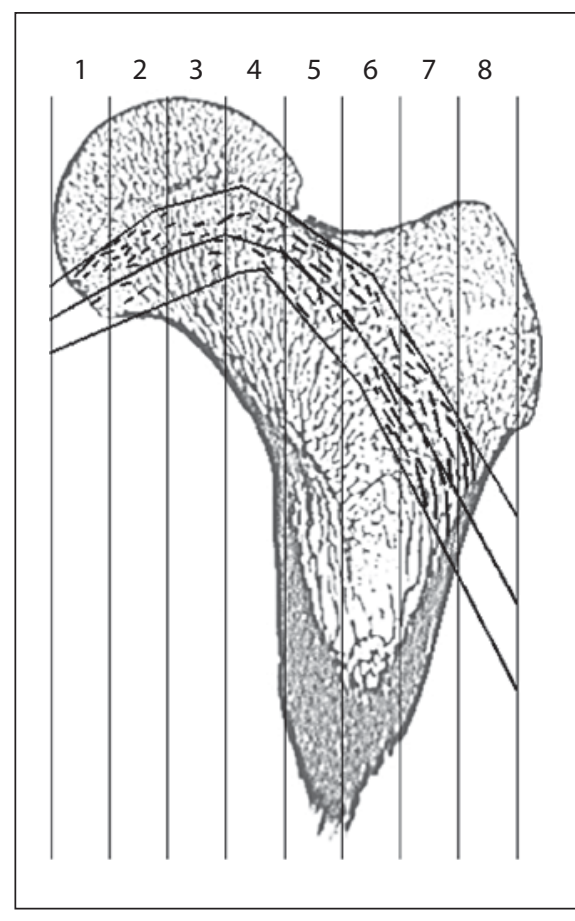

3

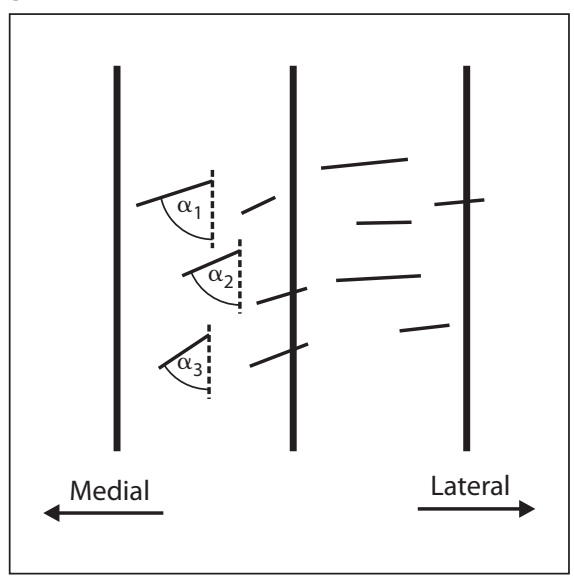

$1 \mathrm{~cm}$ in width. Within every section, the arcuate system was examined in a cranial and a caudal half permitting the detection of differences in the course of the trabecular system from cranial to caudal (fig. 2). All mediocaudally orientated angles between the marked trabeculae and the vertical were measured in every single section thus generated (fig. 3). Thereafter, the mean of all these angles was calculated for each section separately. Then, applying this mean angle to the vertical, for each section a straight line was plotted into the construction, with the line crossing the midpoint of the corresponding lateral edge of the according section. Thus, this straight line could be regarded as a representation of the mean trabecular course within and of the position of each section. We identified the intersection points of each straight line representing the mean trabecular angle of a section with corresponding straight lines of the adjacent sections. Finally, these intersection points served as nodal points through which a spline could be created using the spline function of Allplan. A spline is defined as a curveshaped interpolation of single nodal points; within every interval, this curve is defined by a polynomial of the order $\leq 3$. As the arcuate trabecular system is in fact curvilinear and a spline provides the advantage that the resulting curve is progressing as smoothly as possible through the nodal points (i.e. the edges at the intersection points are merely flattened), this method enabled us to obtain a curve highly concordant with the anatomical original.

Based on all the splines constructed, a resulting spline was calculated (likewise by calculating the mean of corresponding angles) and subsequently plotted in a standard femur mask.

\section{Approximation of the Medial and Trochanteric Trabecular} System

To perform a complete examination of the trabecular architecture of the proximal femur, all angles between the trabeculae of the medial and the arcuate, and the trochanteric and the arcuate trabecular system were measured. Subsequently, the means of these angles were calculated. This was done firstly for each $1-\mathrm{cm}$ section separately, again to be able to detect possible differences in the structural relation of the trabecular systems from medial to lateral; then, the mean of all identifiable angles between trabeculae of the medial and the arcuate, respectively the trochanteric and the arcuate trabecular system was calculated.

\section{Identification of the Structural Relation of the Medial}

Trabecular System and the Epiphysis of the Femoral Head

We were interested in the structural relation between the medial trabecular system and the epiphysis of the femoral head. The epiphysis of the femoral head was well preserved and detectable as an epiphyseal line in the majority of CT scans. The epiphyseal line was marked manually, and then all intersections between the trabeculae of the medial trabecular system and the cranial border of the epiphyseal line were identified. Subsequently, all mediocaudally orientated angles of these intersections were measured and the mean of these angles was calculated.

\section{Results}

\section{Arcuate Trabecular System}

Concerning the arcuate trabecular system, a total of 4,839 single trabeculae could be identified in the CT scans investigated. Figure 4 shows two typical CT scans with identified trabeculae of the arcuate trabecular system and two CT scans with corresponding resulting cra- 


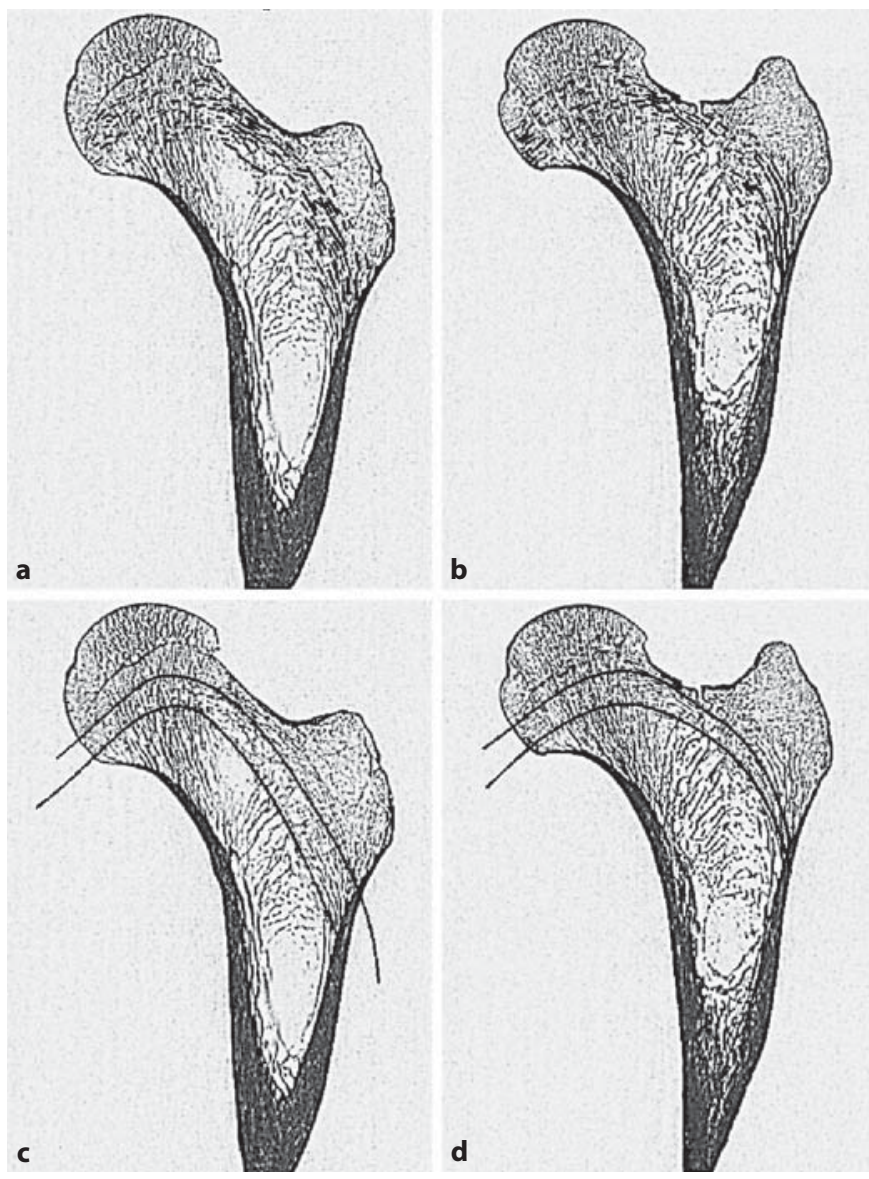

Fig. 4. Two CT scans with identified trabeculae of the arcuate trabecular system. a Proximal femur with valgic tendency. b Proximal femur with varic tendency. c, d Resulting splines representing the course of the arcuate trabecular system.

nial and caudal splines. Due to the fact that all resulting cranial and caudal splines clearly showed concordant overall appearance, all angles measured between each individual trabecula and the vertical were combined and a cumulative spline could be constructed revealing the mean course of all arcuate trabecular systems analyzed using all the CT scans.

\section{Medial and Trochanteric Trabecular Systems}

1,794 intersection points between trabeculae of the medial and the arcuate trabecular system and 846 intersection points between trabeculae of the trochanteric and the arcuate trabecular system could be identified. The corresponding angles revealed the following arithmetic means and standard deviations (SD; coefficients of variation, $\mathrm{CV}$, are shown in parentheses): $92.8 \pm 20.1^{\circ}(21.7 \%)$ in section $1,91.9 \pm 13.9^{\circ}(15.1 \%)$ in section $2,93.0 \pm$ $13.6^{\circ}(14.6 \%)$ in section $3,87.9 \pm 14.8^{\circ}(16.8 \%)$ in section 4 and $84.6 \pm 19.2^{\circ}(22.7 \%)$ in section 5 (regarding intersection points between the medial and arcuate trabecular system), and $83.4 \pm 20.2^{\circ}(24.2 \%)$ in section $5,85.4 \pm$ $16.2^{\circ}(19.0 \%)$ in section $6,86.5 \pm 19.4^{\circ}(22.4 \%)$ in section $7,80.9 \pm 18.6^{\circ}(23.0 \%)$ in section 8 and $86.1 \pm 14.5^{\circ}$ (16.8\%) in section 9 (regarding intersection points between the trochanteric and arcuate trabecular system; fig. 5). With respect to all the angles identified between the trabeculae of the medial and the arcuate trabecular system, a mean angle of $90.7^{\circ}$ (SD: $14.9^{\circ}$; CV: $16.4 \%$ ) and regarding all the angles between the trabeculae of the trochanteric and the arcuate trabecular system, a mean angle of $84.9^{\circ}$ (SD: $18.6^{\circ}$; CV: $21.9 \%$ ) could be calculated.

\section{Scheme of the Trabecular Architecture of the Proximal Femur Resulting from the Angle between the Medial Trabecular System and the Epiphyseal Plate of the Femoral Head}

Considering all 567 identified intersection points between the trabeculae of the medial trabecular system and the epiphyseal plate of the femoral head, the mean of all corresponding mediocaudally orientated angles is $94.7^{\circ}$ (SD: $16.2^{\circ}$; CV: $17.1 \%$ ). Consequently, it is assumed that the medial trabecular system is not only orientated perpendicular to the arcuate system but also to the epiphyseal plate. Based on our results, a scheme of the trabecular architecture of the proximal femur could be set up (fig. 6).

\section{Discussion}

\section{Study Method}

This study was performed to reveal the actual course of the three dominant trabecular systems of the proximal femur by means of a method based on the analysis of CT scans. The trabecular architecture of the proximal human femur has been discussed extensively in the literature. However, in the majority of cases, the morphology is only roughly described, exact measurements of single trabeculae are not performed. The trabeculae are summed up in trabecular systems, which are equal to our assumed trabecular systems, albeit the trochanteric trabecular system included in our analysis has never been further addressed to our knowledge [Singh et al., 1970; Osborne et al., 1980; Martens et al., 1983; Elke et al., 1995; Stiehl et al., 2007]. All femora were suitable according to the method applied: all specimens were of comparable size exhibiting centrum-collum-diaphysis angles adequate to age without 
Fig. 5. Histograms showing the normal distribution of the angles between the trabeculae of the medial and arcuate trabecular system (mean angle $90.7^{\circ}, \mathrm{SD} 14.9^{\circ}, \mathrm{CV}$ $16.4 \%$; a) and the angles between the trochanteric and arcuate trabecular system (mean angle $84.9^{\circ}$, SD $18.6^{\circ}, \mathrm{CV} 21.9 \%$; b).
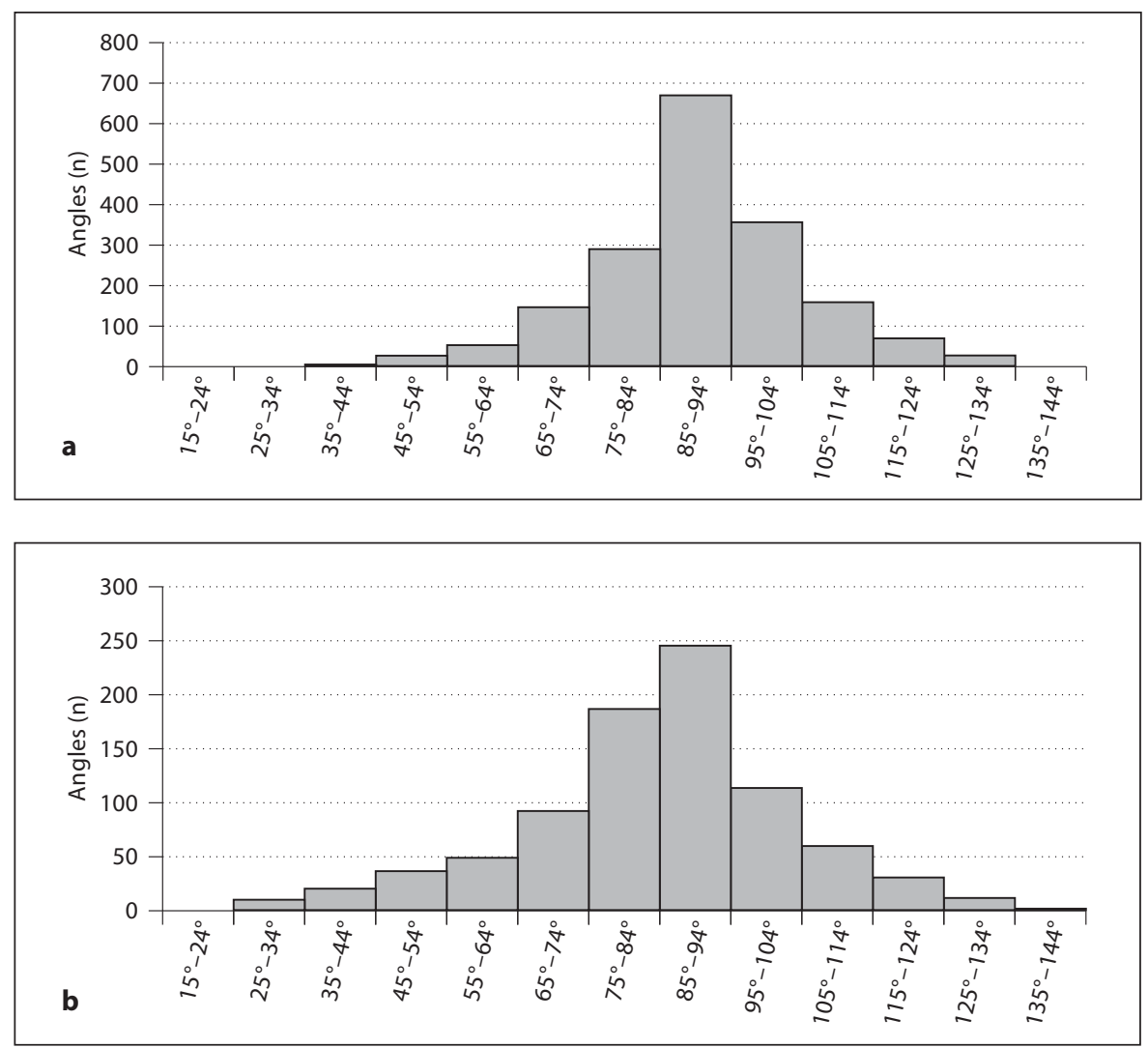

signs of pathological changes. With respect to the identification and marking of the individual trabeculae, it could be criticized that within one trabecula various straight lines can be supposed and therefore, due to a certain examiner dependence, specific orientations could be favored. To eliminate this problem, the straight lines were concurrently positioned along the central axis of the trabeculae, but nevertheless a certain degree of variability is undeniable. However, the differences in these orientations are merely marginal. One aspect regarding the marking of trabeculae needs however improvement: in our study, irrespective of their overall length, all identifiable trabeculae were considered and contributed equally to the arithmetic mean. An algorithm should be developed to take these differences in length into account, and a lower limit of trabecula length should be defined below which no trabeculae are considered. The identification of the outlines of the arcuate trabecular system on the basis of each CT scan also needs critical reconsideration. Although necessary for the definition of cranial and caudal sections, this provided a potential source of bias. Within the femoral head, the arcuate system does not exceed the epiphy- seal plate [Elke et al., 1995]. Consequently, the cranial outline presented slightly distally to the epiphyseal plate of the femoral head and simultaneously in the range of the apophyseal plate of the greater trochanter. The caudal outline was identified where hardly any trabeculae of the arcuate system could be marked. With respect to the method applied, this is the most reasonable technique. More sophisticated analysis devices, e.g. graphical computer programs calculating bone tissue density based on pixel analysis, would not have been able to distinguish to which of the different trabecular systems each single trabecula belongs, predominantly at intersecting regions. According to the method used in this study, only comparable angles (invariably the mediocaudally oriented angles) between each trabecula and the vertical were used for the calculation of the arithmetic mean. Hence, the resulting spline represents the mean course of all arcuate trabecular systems analyzed and provides an excellent picture of the architecture of this trabecular system in the frontal plane. The medial and trochanteric trabecular systems obtained are depicted merely by lines, since only the orientations of this trabecular systems to the arcuate sys- 


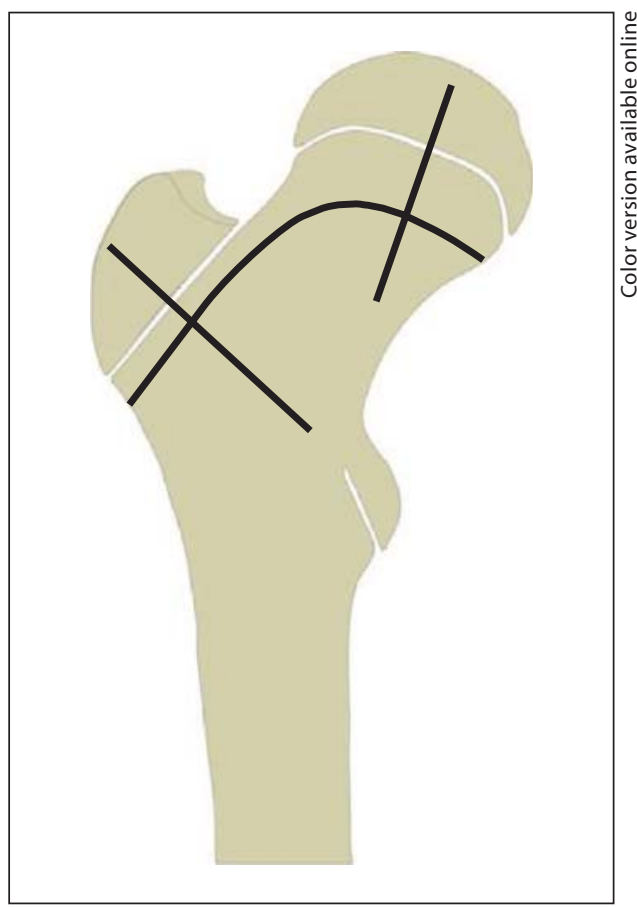

Fig. 6. Resulting scheme of the trabecular architecture of the human proximal femur according to the results presented in this study. Due to historic reasons, the results are represented in a femur mask representing the right human femur.

tem are of interest with respect to subsequent biomechanical interpretations. The present analysis of the trabecular system of the proximal femur was based solely on the frontal plane, a third dimension was not considered and even could not have been analyzed using the method applied in our study. However, since the aim of this study was to compare the biomechanical hypothesis of Pauwels with that of Heimkes, both of which are two-dimensional models, our analysis seemed appropriate. Surely, examiner dependence might be a limitation of the study using the method applied, but it provides the vital advantage that each step of the trabecular analysis can be critically controlled. Other trabecular analysis methods described in the literature either do not provide the possibility to examine each single trabecula of the trabecular systems [Elke et al., 1995; Uchiyama et al., 1999; Stiehl et al., 2007], are predominantly developed for mechanical analyses with limited suitability for structural examinations [Mullender et al., 1998; Homminga et al., 2002; Van Rietbergen et al., 2003], or would have ensued immense complexity and costs [Brismar et al., 1999]. The method used in this study is a simple, innovative technique for the investiga-

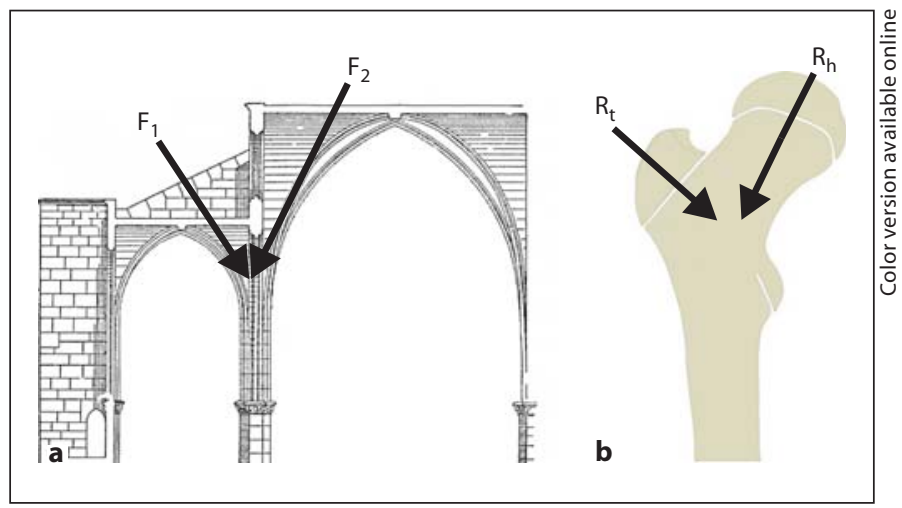

Fig. 7. Heimkes assumes two compression forces acting on the proximal femur: $\mathrm{R}_{\mathrm{h}}$ acting on the femoral head and $\mathrm{R}_{\mathrm{t}}$ acting on the greater trochanter $(\mathbf{b})$. As this concept resembles the force pattern of a clustered pillar in gothic cathedral architecture (a), in this study the hypothesis of Heimkes is referred to as the 'clustered-pillar concept'.

tion of the trabecular structure in the cancellous bone; the results can serve as a basis for the reasonable and specific application of more complex and costly procedures.

The newly measured trabecular structure is used as a basis for biomechanical considerations. As mentioned in the introduction, the alignment of trabeculae with main stresses in the cancellous bone, a concept accepted by the majority of researchers, provides the crucial theoretical basis for this approach.

Although it is not a technique measuring force directly, analysis of the trabecular structure in the cancellous bone in summary provides a smart way to compare existing biomechanical models with a history of stress influence on bone tissue, and the probability of suggested biomechanical models can be determined physiologically.

\section{Biomechanical Interpretation of the Apparent}

Trabecular Structure within the Proximal Femur

The newly identified course of the arcuate trabecular system within the proximal femur and its structural relation to the medial and trochanteric systems provides new insights regarding forces acting on the proximal femur, enabling the comparison between the biomechanical models of Pauwels and Heimkes. Of note, both hypotheses are highly similar, but a closer look at the model of Heimkes reveals that it is merely an amendment to that of Pauwels. Both authors use the one-legged stance as a basis for their biomechanical approach. Indeed, this allows conclusions with respect to the phase of the standing 
leg during slow walking [Bergmann et al., 2001], although inertia forces are neglected. Both models correlate regarding the force acting on the femoral head. The main difference between these two hypotheses is the analysis of the forces affecting the greater trochanteric region. Pauwels oversimplifies the actual situation by considering only the body weight acting on the hip and the muscle forces of the abductors inserting at the greater trochanter [Simoes et al., 2000; Szivek et al., 2000]. In fact, the greater trochanter also serves as the site of origin of the femoral knee extensors (predominantly the vastus lateralis muscle). Acting in concert with the abductors, a counteracting muscle loop is formed. This is most relevant for biomechanical considerations. Pauwels also ignores the existence of an apophyseal plate at the greater trochanter: considering this apophysis and the fact that no case of isolated avulsion of the greater trochanter owing to muscular traction has been described so far, a mere abducting force at the greater trochanter seems highly unlikely.

With respect to the femoral head, there is consensus in the literature that the trabecular system, which proceeds from craniomedial to caudolateral (i.e. the medial trabecular system), is a structural consequence of the body weight acting on the femoral head. Hence, this structure represents a compression load [Singh et al., 1970; Osborne et al., 1980; Martens et al., 1983; Elke et al., 1995; Cody et al., 1996; Birnbaum et al., 2004; Stiehl et al., 2007]. According to the results presented here, this medial trabecular system is orientated approximately perpendicular to both the arcuate trabecular system (mean angle $90.7^{\circ}$ ) and the epiphyseal plate of the femoral head (mean angle $94.7^{\circ}$ ). This finding of an orthogonal orientation of the medial trabecular system to the epiphyseal plate is consistent with a study by Stiehl et al. [2007]. The widely accepted principle that trabeculae align with main forces acting on the cancellous bone may lead to the conclusion that a compression load acts roughly perpendicular to the epiphyseal plate. The analysis of the intersection region between the medial and arcuate trabecular system, which is located directly distal to the epiphyseal plate, likewise revealed an orthogonal orientation of the trabeculae of the two trabecular systems.

Compression of a sample material in one direction simultaneously causes expansion of the material perpendicular to the direction of the compression force. This basic principle of material mechanics is described by Poisson's ratio [Popov, 1978]. We assume that the compression force acting on the femoral head likewise produces stress perpendicular to its direction. On a cellularmolecular basis, this stress can act as a mechanical stim- ulus for osteoblastic differentiation, osteogenic gene expression and finally osteogenesis [Koike et al., 2005; Rath et al., 2008]. The trabeculae of the arcuate trabecular system, which are approximately perpendicular to those of the medial trabecular system, can be regarded as the biological equivalent of this stress perpendicular to the main compressive force.

Analysis of the trabecular system in the lateral proximal femur (region of the greater trochanter) reveals a structure that is also very similar to that within the femoral head. The trochanteric trabecular system firstly identified and analyzed in this study is also orientated approximately perpendicular to the arcuate trabecular system. The similarity of the cancellous structure to that within the femoral head highly suggests a compression load acting on the greater trochanter orientated in mediocaudal direction. Again we assume that the trabeculae of the arcuate trabecular system, which are approximately perpendicular to those of the trochanteric trabecular system, reflect stress orthogonal to the compressive force acting on the greater trochanter that serves as a mechanical stimulus for bone formation.

The existence of the trochanteric system merely reflects a loading of the greater trochanter in mediocaudal direction, either in the form of compression or tension. However, the similarity to the cancellous architecture of the femoral head and the finding of a trabecular system that is approximately perpendicular to the trochanteric trabecular system highly suggest a compression of the greater trochanter in mediocaudal direction, as considered in the model by Heimkes.

Due to the similarity of the force pattern assumed by Heimkes and the forces acting on special columns in gothic cathedral architecture, the theory of Heimkes could be referred to as the 'clustered-pillar concept' (fig. 7).

References

Aamodt, A., J. Lund-Larsen, J. Eine, E. Andersen, P. Benum, O.S. Husby (1997) In vivo measurements show tensile axial strain in the proximal lateral aspect of the human femur. J Orthop Res 15: 927-931.

Bergmann, G., G. Deuretzbacher, M. Heller, F. Graichen, A. Rohlmann, J. Strauss, G.N. Duda (2001) Hip contact forces and gait patterns from routine activities. J Biomech 34 : 859-871.

Berzins, A., D.R. Sumner, T.P. Andriacchi, J.O. Galante (1993) Stem curvature and load angle influence the initial relative bone-implant motion of cementless femoral stems. J Orthop Res 11: 758-769. 
Biewener, A.A., N.L. Fazzalari, D.D. Konieczynski, R.V. Baudinette (1996) Adaptive changes in trabecular architecture in relation to functional strain patterns and disuse. Bone 19: $1-8$.

Birnbaum, K., C.H. Siebert, T. Pandorf, E. Schopphoff, A. Prescher, F.U. Niethard (2004) Anatomical and biomechanical investigations of the iliotibial tract. Surg Radiol Anat 26: 433-446.

Birnbaum, K., R. Sindelar, J.R. Gartner, D.C. Wirtz (2001) Material properties of trabecular bone structures. Surg Radiol Anat 23: 399-407.

Brand, R.A., D.R. Pedersen, D.T. Davy, G.M. Kotzar, K.G. Heiple, V.M. Goldberg (1994) Comparison of hip force calculations and measurements in the same patient. J Arthroplasty 9: 45-51.

Brismar, T.B., M. Karlsson, T.Q. Li, H. Ringertz (1999) Orientation of trabecular bone in human vertebrae assessed by MRI. Eur Radiol 9: 643-647.

Buhler, D.W., U. Berlemann, K. Lippuner, P. Jaeger, L.P. Nolte (1997) Three-dimensional primary stability of cementless femoral stems. Clin Biomech (Bristol, Avon) 12: $75-$ 86.

Carter, D.R., T.E. Orr, D.P. Fyhrie (1989) Relationships between loading history and femoral cancellous bone architecture. J Biomech 22: 231-244.

Cody, D.D., D.A. McCubbrey, G.W. Divine, G.J. Gross, S.A. Goldstein (1996) Predictive value of proximal femoral bone densitometry in determining local orthogonal material properties. J Biomech 29: 753-761.

Cristofolini, L., M. Juszczyk, S. Martelli, F. Taddei, M. Viceconti (2007) In vitro replication of spontaneous fractures of the proximal human femur. J Biomech 40: 2837-2845.

Duda, G.N., M. Heller, J. Albinger, O. Schulz, E. Schneider, L. Claes (1998) Influence of muscle forces on femoral strain distribution. J Biomech 31: 841-846.

Elke, R.P., E.J. Cheal, C. Simmons, R. Poss (1995) Three-dimensional anatomy of the cancellous structures within the proximal femur from computed tomography data. J Orthop Res 13: 513-523.

Fyhrie, D.P., D.R. Carter (1986) A unifying principle relating stress to trabecular bone morphology. J Orthop Res 4: 304-317.

Gebauer, D., H.J. Refior, M. Haake (1989) Micromotions in the primary fixation of cementless femoral stem prostheses. Arch Orthop Trauma Surg 108: 300-307.

Gomberg, B.R., P.K. Saha, F.W. Wehrli (2003) Topology-based orientation analysis of trabecular bone networks. Med Phys 30: 158168

Gotze, C., W. Steens, V. Vieth, C. Poremba, L. Claes, J. Steinbeck (2002) Primary stability in cementless femoral stems: custom-made versus conventional femoral prosthesis. Clin Biomech (Bristol, Avon) 17: 267-273.
Heimkes, B., P. Posel, W. Plitz, V. Jansson (1993) Forces acting on the juvenile hip joint in the one-legged stance. J Pediatr Orthop 13: 431436.

Hêrt, J. (1994) A new attempt at the interpretation of the functional architecture of the cancellous bone. J Biomech 27: 239-242.

Homminga, J., B.R. McCreadie, T.E. Ciarelli, H. Weinans, S.A. Goldstein, R. Huiskes (2002) Cancellous bone mechanical properties from normals and patients with hip fractures differ on the structure level, not on the bone hard tissue level. Bone 30: 759-764.

Kerr, R., D. Resnick, D.J. Sartoris, S. Kursunoglu, C. Pineda, P. Haghighi, G. Greenway, J. Guerra, Jr. (1986) Computerized tomography of proximal femoral trabecular patterns. J Orthop Res 4: 45-56.

Kinney, J.H., J.S. Stolken, T.S. Smith, J.T. Ryaby, N.E. Lane (2005) An orientation distribution function for trabecular bone. Bone 36: $193-$ 201.

Koike, M., H. Shimokawa, Z. Kanno, K. Ohya, K. Soma (2005) Effects of mechanical strain on proliferation and differentiation of bone marrow stromal cell line ST2. J Bone Miner Metab 23: 219-225.

Maher, S.A., P.J. Prendergast (2002) Discriminating the loosening behaviour of cemented hip prostheses using measurements of $\mathrm{mi}$ gration and inducible displacement. J Biomech 35: 257-265.

Martens, M., R. Van Audekercke, P. Delport, P. De Meester, J.C. Mulier (1983) The mechanical characteristics of cancellous bone at the upper femoral region. J Biomech 16: 971983.

McKellop, H., E. Ebramzadeh, P.G. Niederer, A Sarmiento (1991) Comparison of the stability of press-fit hip prosthesis femoral stems using a synthetic model femur. J Orthop Res 9: 297-305.

Meyer, G.H. (1867) Die Architektur der Spongiosa. Arch Anat Physiol Wiss Med 34: 615628.

Mikic, B., D.R. Carter (1995) Bone strain gage data and theoretical models of functional adaptation. J Biomech 28: 465-469.

Mullender, M., B. van Rietbergen, P. Ruegsegger, R. Huiskes (1998) Effect of mechanical set point of bone cells on mechanical control of trabecular bone architecture. Bone 22: 125 131.

Osborne, D., E. Effmann, K. Broda, J. Harrelson (1980) The development of the upper end of the femur, with special reference to its internal architecture. Radiology 137: 71-76.

Pauwels, F. (1935) Der Schenkelhalsbruch - Ein mechanisches Problem - Grundlagen des Heilungsvorganges. Prognose und kausale Therapie. Z Orthop Chir Suppl 63: 38-43.
Pauwels, F. (1954) Über die Verteilung der Spongiosadichte im coxalen Femurende und ihre Bedeutung für die Lehre vom funktionellen Bau des Knochens. Siebenter Beitrag zur funktionellen Anatomie und kausalen Morphologie des Stützapparates. Morph Jb 95: 35-54.

Pedersen, D.R., R.A. Brand, D.T. Davy (1997) Pelvic muscle and acetabular contact forces during gait. J Biomech 30: 959-965.

Popov, E.P. (1978) Mechanics of Materials, ed 2. Englewood Cliffs, Prentice Hall.

Rath, B., J. Nam, T.J. Knobloch, J.J. Lannutti, S. Agarwal (2008) Compressive forces induce osteogenic gene expression in calvarial osteoblasts. J Biomech 41: 1095-1103.

Rudman, K.E., R.M. Aspden, J.R. Meakin (2006) Compression or tension? The stress distribution in the proximal femur. Biomed Eng Online $5: 12$.

Ruimerman, R., B. Van Rietbergen, P. Hilbers, R. Huiskes (2003) A 3-dimensional computer model to simulate trabecular bone metabolism. Biorheology 40: 315-320.

Rydell, N. (1973) Biomechanics of the hip-joint. Clin Orthop Relat Res 92: 6-15.

Simoes J.A., M.A. Vaz, S. Blatcher, M. Taylor (2000) Influence of head constraint and muscle forces on the strain distribution within the intact femur. Med Eng Phys 22: 453-459.

Singh, M., A.R. Nagrath, P.S. Maini (1970) Changes in trabecular pattern of the upper end of the femur as an index of osteoporosis. J Bone Joint Surg Am 52: 457-467.

Smit, T.H., A. Odgaard, E. Schneider (1997) Structure and function of vertebral trabecular bone. Spine 22: 2823-2833.

Stiehl, J.B., D. Jacobson, G. Carrera (2007) Morphological analysis of the proximal femur using quantitative computed tomography. Int Orthop 31: 287-292.

Szivek, J.A., J.B. Benjamin, P.L. Anderson (2000) An experimental method for the application of lateral muscle loading and its effect on femoral strain distributions. Med Eng Phys 22: 109-116.

Taylor, M.E., K.E. Tanner, M.A. Freeman, A.L. Yettram (1996) Stress and strain distribution within the intact femur: compression or bending? Med Eng Phys 18: 122-131.

Turner, C.H., V. Anne, R.M. Pidaparti (1997) A uniform strain criterion for trabecular bone adaptation: do continuum-level strain gradients drive adaptation? J Biomech 30: 555563.

Uchiyama, T., T. Tanizawa, H. Muramatsu, N. Endo, H.E. Takahashi, T. Hara (1999) Threedimensional microstructural analysis of human trabecular bone in relation to its mechanical properties. Bone 25: 487-491.

Van Rietbergen, B., R. Huiskes, F. Eckstein, P. Ruegsegger (2003) Trabecular bone tissue strains in the healthy and osteoporotic human femur. J Bone Miner Res 18: 17811788 